\title{
Inequality of Mean Interdivision Time and Doubling Time
}

\author{
By P. R. PAINTER AND A. G. MARR \\ Department of Bacteriology, University of California, \\ Davis, California 95616, U.S.A.
}

(Accepted for publication 27 February 1967)

If bacteria are watched from the time they are formed until they divide their interdivision times $\tau$ can be measured, and the distribution of these times will be given by the frequency function $f(\tau)$. The mean interdivision time $\bar{\tau}$ is then

$$
\int_{0}^{\infty} \tau f(\tau) d \tau
$$

Powell (1956) pointed out that the equation

$$
\bar{\tau}=\ln 2 / k,
$$

in which the specific growth rate is denoted by $k$ and $\ln 2 / k$ is the doubling time of the population, is usually false. However, several subsequent investigators have continued to assume this specious equality. In this note we prove the inequality

$$
\bar{\tau}>\ln 2 / k
$$

and derive the approximation formula

$$
\bar{\tau} \approx \ln 2 / k+k \sigma^{2} / 2
$$

in which $\sigma$ is the standard deviation of $f(\tau)$. We make a few applications of (1) and (2) to the analysis of experimental and theoretical results.

If the interdivision times of individual bacteria are independent, it may be shown from renewal theory (Feller, 1941) that $2 e^{-k r} f(\tau)$ is also a frequency function (Powell, 1956; Harris, 1959;); i.e.

$$
\int_{0}^{\infty} 2 e^{-k \tau} f(\tau) d \tau=1
$$

Interdivision times are known not to be independent. The interdivision times of sisters are positively correlated (Powell, 1955; Schaechter, Williamson, Hood \& Koch, 1962; Kubitschek, 1962), and the interdivision times of mother and daughter are negatively correlated (Powell, 1955). However, equation (3) can be derived without the assumption of independence.

If a bacterial population consisting of $N(0)$ cells is in balanced growth, and if $N(0)$ is sufficiently large so that the number of viable cells $N(t)$ at some time, $t$, can be written as

$$
N(\dot{t})=N(0) e^{k t}
$$

with negligible error, and if $f(\tau)$ is independent of time, one can account for all of the cells present at time $T$. One portion, $R$, consists of cells present at time 0 which have not yet divided; the remainder are new cells produced by divisions in the interval 
$[0, T]$. At any intermediate time $t(0 \leqslant t \leqslant T)$ the rate of formation of cells that will be extant at time $T$ is

$$
2 k N(0) e^{k t} \int_{T-t}^{\infty} f(\tau) d \tau
$$

The number of new cells formed in the interval $[0, T]$ which are extant at time $T$ is

Substituting $t=T-x$ gives

$$
\int_{0}^{T} \int_{T-t}^{\infty} 2 k N(0) e^{k t} f(\tau) d \tau d t
$$

$$
\int_{0}^{T} \int_{x}^{\infty} 2 k N(0) e^{k(T-x)} f(\tau) d \tau d x .
$$

Consequently, at time $T$ the total number of cells is

or

$$
\begin{gathered}
N(0) e^{k T}=R+N(0) e^{k T} \int_{0}^{T} \int_{x}^{\infty} 2 k e^{-k x} f(\tau) d \tau d x \\
\int_{0}^{T} \int_{x}^{\infty} 2 k e^{-k x} f(\tau) d \tau d x=1-[R / N(0)] e^{-k T} .
\end{gathered}
$$

As $T$ approaches infinity, the term $[R / N(0)] e^{-k T}$ approaches zero giving

$$
\int_{0}^{\infty} \int_{x}^{\infty} 2 k e^{-k x} f(\tau) d \tau d x=1 .
$$

Interchanging the order of integration gives

$$
\int_{0}^{\infty} \int_{0}^{\tau} 2 k e^{-k x} f(\tau) d x d \tau=1
$$

which, upon performing the integration with respect to $x$, gives equation (3).

With the assumptions and approach used in this derivation it may be demonstrated that the frequency function of interdivision times of dividing cells is $2 e^{-k \tau} f(\tau)$ (Powell's (1956) carrier distribution, $C(\tau)$ ). It may also be shown that the frequency function of interdivision times of all extant cells is $2\left(1-e^{-k t}\right) f(\tau)$ (Powell's (1964) $P(\tau)$ ).

Factoring $2 e^{-k \bar{\tau}}$ from equation (3) gives

$$
2 e^{-k \bar{\tau}} \int_{0}^{\infty} e^{-k(\tau-\bar{\tau})} f(\tau) d \tau
$$

The inequality,

$$
1-x<e^{-x}
$$

holds for all $x \neq 0$. Since analysis and experiment demonstrate some dispersion of interdivision time (Powell, $[1955$; Kubitschek, 1962), we write the inequality

$$
2 e^{-k \bar{\tau}} \int_{0}^{\infty}[1-k(\tau-\bar{\tau})] f(\tau) d \tau<1 .
$$

Using the definition of $\bar{\tau}$, we have by integration

and, hence,

$$
\begin{gathered}
2 e^{-k \bar{\tau}}<1 \\
\bar{\tau}>\ln 2 / k .
\end{gathered}
$$

Formula (2) can be derived by expressing the integral in equation (4) as

$$
\int_{0}^{\infty} e^{-k(\tau-\bar{\tau})} f(\tau) d \tau=1+\sum_{i=1}^{\infty} \mu_{i}(-k)^{i} / i !
$$


in which $\mu_{i}$ is the $i$ th central moment of $f(\tau)$. Equation (5) is valid if all of the moments exist and the series converges. For many types of functions the terms of the series in (5) rapidly go to zero. If all terms beyond the second are ignored and the substitution is made for the integral in (4), we obtain

$$
2 e^{-k \bar{\tau}}\left(1-\mu_{1} k+\mu_{2} k^{2} / 2\right) \approx 1 \text {. }
$$

Since $\mu_{1}=0$ and $\mu_{2}=\sigma^{2}$, we have

$$
1+\sigma^{2} k^{2} / 2 \approx e^{k \bar{\tau}} / 2 .
$$

If the quantity $\sigma^{2} k^{2} / 2$ is much less than 1 ,

$$
\ln \left(1+\sigma^{2} k^{2} / 2\right) \approx \sigma^{2} k^{2} / 2,
$$

since

$$
\ln (1+x)=\sum_{n=1}^{\infty}(-1)^{n+1} x^{n} / n \text { for }|x|<1 \text {. }
$$

Taking logarithms of equation (6) and substituting the approximation above gives

or

$$
\begin{aligned}
& \sigma^{2} k^{2} / 2 \approx k \bar{\tau}-\ln 2 \\
& \bar{\tau} \approx \ln 2 / k+k \sigma^{2} / 2 .
\end{aligned}
$$

Formula (2) has been derived for the special case that $f(\tau)$ is a uniform distribution (Engleberg, 1964) or a Gaussian distribution (Barrett, 1966). If some information is given about $f(\tau)$, it is often practicable to use the extended law of the mean to establish a limit to the error in (2).

The approximation by formula (2) was tested by a numerical method using a digital computer. Powell (1955) has found a good fit of experimental measurements of $f(\tau)$ to a Pearson Type 3 distribution. The frequency function of this distribution with a mean of 1 was computed for various standard deviations. For each distribution the value of $k$ was computed by successive approximation using equation (3) and integrating by Simpson's rule. The values of $k$ are accurate to \pm 0.00001 . For the special case that $f(\tau)$ is a Pearson Type 3 distribution, Powell (1956) has shown that

$$
k=\left(2^{1 / 0}-1\right) / m \text {, }
$$

where $\tau=m g$ and $\sigma^{2}=m^{2} g$. The stated accuracy of the general numerical approximation of $k$ was verified by direct solution. The values of $\bar{\tau}$ were estimated by formula (2).

Table 1 shows that the approximation $\bar{\tau}=\ln 2 / k$ is 'good only for small values of $\sigma / \bar{\tau}$. When $\sigma / \bar{\tau}=0.2$, the error is less than $2 \%$; but when $\sigma / \bar{\tau}=0.5$, the error is nearly $10 \%$. The error in estimating $\bar{\tau}$ by formula (2) increases as $\sigma$ increases. The error of estimation at large $\sigma$ results from a significant contribution by higher moments of the $\tau$-distribution. At $\sigma=0.5$, the error is approximately $1 \%$. Since the coefficient of variation of interdivision time $(\sigma / \bar{\tau})$ rarely exceeds 0.5 , the approximation by formula (2) is sufficiently accurate for most purposes. A better approximation can be made by including terms which correspond to the third and fourth moments, but the formula becomes quite complicated.

Table 2 compares the estimates of $\bar{\tau}$ with experimental results of Powell (1955). The agreement is satisfactory. To our knowledge, no other measurements have been made with sufficient precision to establish the discrepancy between mean interdivision time and doubling time.

Relation (1) can be used to analyse parts of Powell's (1964) note on Koch and Schaechter's hypothesis. By assuming exponential growth of individuals and equal 
Table 1. Estimation of mean interdivision time from specific growth rate and standard deviation of $f(\tau)$

$\begin{array}{cccc}\begin{array}{c}\text { Standard } \\ \text { deviation } \\ (\sigma)\end{array} & \begin{array}{c}\text { Specific } \\ \text { growth rate } \\ (k)^{*}\end{array} & \begin{array}{c}\text { Doubling } \\ \text { time } \\ (\ln 2 / k)\end{array} & \begin{array}{c}\text { Estimated mean } \\ \text { interdivision } \\ \text { time }(\tau) \dagger\end{array} \\ 0.00 & - & 1.00000 & 1.00000 \\ 0.05 & 0.69375 & 0.99914 & 1.00001 \\ 0.06 & 0.69401 & 0.99876 & 1.00001 \\ 0.07 & 0.69433 & 0.99831 & 1.00001 \\ 0.08 & 0.69469 & 0.99778 & 1.00000 \\ 0.09 & 0.69510 & 0.99719 & 1.00001 \\ 0.10 & 0.69556 & 0.99654 & 1.00002 \\ 0.20 & 0.70285 & 0.98620 & 1.00026 \\ 0.30 & 0.71522 & 0.96914 & 1.00135 \\ 0.40 & 0.73304 & 0.94558 & 1.00422 \\ 0.50 & 0.75682 & 0.91787 & 1.01047\end{array}$

* Computed from $\int_{0}^{\infty} 2 e^{-k t} f(\tau) d \tau=1$ assuming $r$ is distributed as a Pearson Type 3 distribution with standard deviation $=\sigma$, and mean $=1 \cdot 00000$.

$\dagger$ Estimated by formula (2).

Table 2. Comparison of estimates of mean interdivision time with the experimental results of Powell (1955)

\begin{tabular}{|c|c|c|c|c|c|}
\hline Organism & $\begin{array}{l}\text { Standard } \\
\text { deviation, } \\
\sigma \text { (min.) }\end{array}$ & $\begin{array}{l}\text { Specific growth } \\
\left.\text { rate, } k \text { (min. }^{-1}\right)\end{array}$ & $\begin{array}{l}\ln 2 / k \\
\text { (min.) }\end{array}$ & $\begin{array}{c}\bar{\tau} \text {, observed } \\
\text { (min.) }\end{array}$ & $\bar{\tau}, \underset{\text { (min.) }}{\text { estimated }}$ \\
\hline $\begin{array}{l}\text { Pseudomonas aeruginosa } \\
\text { Bacillus megaterium } \\
\text { Bacillus mycoides }\end{array}$ & $\begin{array}{l}5 \cdot 37 \\
7 \cdot 76 \\
14 \cdot 2\end{array}$ & $\begin{array}{l}0.01831^{*} \\
0.03259 \dagger \\
0.02637^{*}\end{array}$ & $\begin{array}{l}37 \cdot 85 \\
21 \cdot 27 \\
26 \cdot 29\end{array}$ & $\begin{array}{l}38 \cdot 1 \\
22 \cdot 3 \\
28 \cdot 7\end{array}$ & $\begin{array}{l}38 \cdot 11 \\
22 \cdot 25 \\
28 \cdot 95\end{array}$ \\
\hline
\end{tabular}

* Calculated by Powell (1956) by fitting a Pearson Type 3 distribution to the data and numerically solving $\int_{0}^{\infty} 2^{-k \tau} f(\tau) d \tau=1$ for $k$.

$\dagger$ Calculated by Powell (1956) by plotting the logarithm of number of extant cells versus time.

division, he derives an expression (his equation [4]) for $f(\tau)$ that is symmetrical about $\ln 2 / k$. But this can be true only if $\bar{\tau}=\ln 2 / k$, contradicting (1). His error is the assumption that the frequency function of the size of dividing cells is the same as the frequency function of the size at division of sample of newly formed cells. These functions describe different populations.

The experimental work was supported by Grants ARO-49-092-65-G95 and MD-49-193-65-G160 from the United States Army. Digital computations were financially supported by Grant USPHS AI-05526 from the National Institutes of Health.

\section{REFERENCES}

BARRetT, J. C. (1966). A mathematical model of the mitotic cycles. J. natn. Cancer Inst. $37,443$. ENGLEBERG, J. (1964). The decay of synchronization of cell division. Expl. Cell Res. 36, 647. Feller, W. (1941). On the integral equation of renewal theory. Ann. math. Statist. 12, 243.

HARRIs, T. E. (1959). A mathematical model for multiplication by binary fission. In The Kinetics of Cellular Proliferation, ed. by F. Stohlman, p. 368. New York: Grune and Stratton. 
KUBITSCHEK, H. E. (1962). Normal distribution of cell generation rate. Expl. Cell Res. $26,439$.

Powell, E. O. (1955). Some features of the generation time of individual bacteria. Biometrika, 42, 16.

PowELL, E. O. (1956). Growth rate and generation time of bacteria, with special reference to continuous culture. J. gen. Microbiol. 37, 281.

Powell, E. O. (1964). A note on Koch and Schaechter's hypothesis about growth and fission of bacteria. J. gen. Microbiol. 37, 281.

Schaechter, M., Williamson, J. P., Hood, J. R. \& Koch A. L. (1962). Growth, cell and nuclear division in some bacteria. J. gen. Microbiol. 29, 421. 